\title{
Fluctuation Diagnostics of the Electron Self-Energy: Origin of the Pseudogap Physics
}

\author{
O. Gunnarsson, ${ }^{1}$ T. Schäfer, ${ }^{2}$ J. P. F. LeBlanc, ${ }^{3,4}$ E. Gull, ${ }^{4}$ J. Merino, ${ }^{5}$ G. Sangiovanni, ${ }^{6}$ G. Rohringer, ${ }^{2}$ and A. Toschi ${ }^{2}$ \\ ${ }^{1}$ Max-Planck-Institut für Festkörperforschung, Heisenbergstraße 1, D-70569 Stuttgart, Germany \\ ${ }^{2}$ Institute of Solid State Physics, Vienna University of Technology, A-1040 Vienna, Austria \\ ${ }^{3}$ Max-Planck-Institute for the Physics of Complex Systems, D-01187 Dresden, Germany \\ ${ }^{4}$ Department of Physics, University of Michigan, Ann Arbor, Michigan 48109, USA \\ ${ }^{5}$ Departamento de Física Teórica de la Materia Condensada, IFIMAC Universidad Autónoma de Madrid, Madrid 28049, Spain \\ ${ }^{6}$ Institute of Physics and Astrophysics, University of Würzburg, D-97070 Würzburg, Germany
}

(Received 25 November 2014; published 10 June 2015)

\begin{abstract}
We demonstrate how to identify which physical processes dominate the low-energy spectral functions of correlated electron systems. We obtain an unambiguous classification through an analysis of the equation of motion for the electron self-energy in its charge, spin, and particle-particle representations. Our procedure is then employed to clarify the controversial physics responsible for the appearance of the pseudogap in correlated systems. We illustrate our method by examining the attractive and repulsive Hubbard model in two dimensions. In the latter, spin fluctuations are identified as the origin of the pseudogap, and we also explain why $d$-wave pairing fluctuations play a marginal role in suppressing the low-energy spectral weight, independent of their actual strength.
\end{abstract}

DOI: 10.1103/PhysRevLett.114.236402

PACS numbers: 71.27.+a, 71.10.Fd

Introduction.-Correlated electron systems display some of the most fascinating phenomena in condensed matter physics, but their understanding still represents a formidable challenge for theory and experiments. For photoemission [1] or STM [2,3] spectra, which measure single-particle quantities, information about correlation is encoded in the electronic self-energy $\Sigma$. However, due to the intrinsically many-body nature of the problems, even an exact knowledge of $\Sigma$ is not sufficient for an unambiguous identification of the underlying physics. A perfect example of this is the pseudogap observed in the single-particle spectral functions of underdoped cuprates [4], and, more recently, of their nickelate analogues [5]. Although relying on different assumptions, many theoretical approaches provide self-energy results compatible with the experimental spectra. This explains the lack of a consensus about the physical origin of the pseudogap: In the case of cuprates, the pseudogap has been attributed to spin fluctuations [6-10], preformed pairs [11-15], Mottness $[16,17]$, and, recently, to the interplay with charge fluctuations [18-21] or to Fermi-liquid scenarios [22]. The existence and the role of ( $d$-wave) superconducting fluctuations [11-15] in the pseudogap regime are still openly debated for the basic model of correlated electrons, the Hubbard model.

Experimentally, the clarification of many-body physics is augmented by a simultaneous investigation at the two-particle level, i.e., via neutron scattering [23], infrared or optical [24] and pump-probe spectroscopy [25], muon-spin relaxation [26], and correlation or coincidence two-particle spectroscopies [27-29]. Analogously, theoretical studies of $\Sigma$ can also be supplemented by a corresponding analysis at the two-particle level. In this
Letter, we study the influence of the two-particle fluctuations on $\Sigma$ via its equation of motion. We apply this method of "fluctuation diagnostics" to identify the role played by different collective modes in the pseudogap physics.

Self-energy decomposition.-We emphasize that all concepts and equations below are applicable within any theoretical approach in which the self-energy and the two-particle scattering amplitude are calculated without a priori assumptions of a predominant type of fluctuations. This includes quantum Monte Carlo (QMC) methods (e.g., lattice QMC [30]), functional renormalization group [31], parquet approximation [32-34], and cluster extensions [35] of the dynamical mean field theory (DMFT) $[36,37]$ such as the cellular-DMFT [38,39] or the dynamical cluster approximation (DCA) [40]. Within diagrammatic extensions [41-47] of DMFT, our analysis is applicable if parquet-like diagrams are included [48-50]. The outputs of these techniques can be then post-processed by means of the fluctuation diagnostics with a comparably lower numerical effort [51].

The self-energy describes all scattering effects of one added or removed electron, when propagating through the lattice. In correlated electronic systems, these scattering events originate from the Coulomb interaction among the electrons themselves, rather than from the presence of an external potential. Therefore, $\Sigma$ is entirely determined by the full two-particle scattering amplitude $F$. The formal relation between $F$ and $\Sigma$ is known as the DysonSchwinger equation of motion (EOM) [56]. In the important case of a purely local interaction (as in the Hubbard model [57-59]), this reads (in the paramagnetic phase) 
$\Sigma(k)=\frac{U n}{2}-\frac{U}{\beta^{2} N} \sum_{k^{\prime}, q} F_{\uparrow \downarrow}\left(k, k^{\prime}, q\right) g\left(k^{\prime}\right) g\left(k^{\prime}+q\right) g(k+q)$,

where $U$ is the (bare) Hubbard interaction, $n$ the electronic density, $g$ the electron Green's function, $\beta=1 / T$ the inverse temperature, and $N$ the normalization of the momentum summation [we adopt the notation $k=$ $(\nu, \mathbf{K}) / q=(\omega, \mathbf{Q})$ for the fermionic/bosonic Matsubara frequencies $\nu / \omega$ and momenta $\mathbf{K} / \mathbf{Q}$; see the Supplemental Material [51] for details]. Finally, $F_{\uparrow \downarrow}$ is the full scattering amplitude (vertex) between electrons with opposite spins: It consists of repeated two-particle scattering events in all possible configurations compatible with energy, momentum, or spin conservation. Therefore, it contains the complete information of the two-particle correlations of the system. Yet, much of the information encoded in $F_{\uparrow \downarrow}$ about the specific physical processes determining $\Sigma$ is washed out by averaging over all two-particle scattering events, i.e., by the summations on the right-hand side of Eq. (1). Hence, an unambiguous identification of the physical role played by the underlying scattering or fluctuation processes requires a "disentanglement" of the EOM. The most obvious approach would be a direct decomposition of the full scattering amplitude $F_{\uparrow \downarrow}$ of Eq. (1) in all possible fluctuation channels, the so-called parquet $[32-34,60]$ decomposition. This approach works well in the weakly correlated regime (small $U$, large doping, high $T$ ), whereas for stronger correlations it suffers from intrinsic divergences, recently discovered in the Hubbard and Falicov-Kimball models [61-63]; see also Ref. [64]. For example, in our DCA calculations for the two-dimensional (2D) Hubbard model the breakdown of the parquet decomposition of $\Sigma$ occurs at lower values of $U$ (or larger values of doping) than those for which pseudogap physics is numerically observed $[61,65]$.

In this Letter we present an alternative route that can be followed to circumvent this problem. Our idea exploits the freedom of employing formally equivalent analytical representations of the EOM. For instance, by means of SU(2) symmetry and "crossing relations" (see, e.g., Refs. [66,67]), we can express $F_{\uparrow \downarrow}$ in Eq. (1) in terms of the corresponding vertex functions of the spin or magnetic $F_{\mathrm{sp}}=F_{\uparrow \uparrow}-F_{\uparrow \downarrow}$ and charge or density $F_{\mathrm{ch}}=$ $F_{\uparrow \uparrow}+F_{\uparrow \downarrow}$ sectors. Analogously, a rewriting in terms of the particle-particle sector notation is done via $F_{\mathrm{pp}}\left(k, k^{\prime}, q\right)=$ $F_{\uparrow \downarrow}\left(k, k^{\prime}, q-k-k^{\prime}\right)$. Inserting these results in Eq. (1) and performing variable transformations, we recover Eq. (1), with $F_{\uparrow \downarrow}$ replaced by $F_{\mathrm{sp}}, F_{\mathrm{ch}}$, or $F_{\mathrm{pp}}$ [51]. These three expressions,

$$
\Sigma(k)-\Sigma_{\mathrm{H}}=\frac{U}{\beta^{2} N} \sum_{k^{\prime}, q} F_{\mathrm{sp}}\left(k, k^{\prime} ; q\right) g\left(k^{\prime}\right) g\left(k^{\prime}+q\right) g(k+q),
$$

$$
\begin{aligned}
& =-\frac{U}{\beta^{2} N} \sum_{k^{\prime}, q} F_{\mathrm{ch}}\left(k, k^{\prime} ; q\right) g\left(k^{\prime}\right) g\left(k^{\prime}+q\right) g(k+q), \\
& =-\frac{U}{\beta^{2} N} \sum_{k^{\prime}, q} F_{\mathrm{pp}}\left(k, k^{\prime} ; q\right) g\left(k^{\prime}\right) g\left(q-k^{\prime}\right) g(q-k),
\end{aligned}
$$

yield the same result for $\Sigma$ after all internal summations are performed $\left(\Sigma_{\mathrm{H}}\right.$ denotes the constant Hartree term $\left.U n / 2\right)$. Crucial physical insight can be gained at this stage, by performing partial summations. We can, e.g., perform all summations, except for the one over the transfer momentum $\mathbf{Q}$. This gives $\tilde{\Sigma}_{\mathbf{Q}}(k)$, i.e., the contribution to $\Sigma$ for fixed $\mathbf{Q}$, so that $\Sigma(k)=\sum_{\mathbf{Q}} \tilde{\Sigma}_{\mathbf{Q}}(k)$. The vector $\mathbf{Q}$ corresponds to a specific spatial pattern given by the Fourier factor $e^{i \mathbf{Q} \mathbf{R}_{i}}$. For a given representation such a spatial structure is associated to a specific collective mode, e.g., $\mathbf{Q}=(\pi, \pi)$ for antiferromagnetic or charge-density wave $(\mathrm{CDW})$ and $\mathbf{Q}=(0,0)$ for superconducting or ferromagnetic fluctuations. Hence, if one of these contributions dominates, $\tilde{\Sigma}_{\mathbf{Q}}(k)$ is strongly peaked at the $\mathbf{Q}$ vector of that collective mode, provided that the corresponding representation of the EOM is used. On the other hand, in a different representation, not appropriate for the dominant mode $\tilde{\Sigma}_{\mathbf{Q}}(k)$ will display a weak $\mathbf{Q}$ dependence. These heuristic considerations can be formalized by expressing $F$ through its main momentum and frequency structures $[51,66]$. Hence, in cases where the impact of the different fluctuation channels on $\Sigma$ is not known a priori, the analysis of the $\mathbf{Q}$ dependence of $\tilde{\Sigma}_{\mathbf{Q}}(k)$ in the alternative representations of the EOM will provide the desired diagnostics. Below, we show that this procedure works well for the 2D (attractive and repulsive) Hubbard models, allowing for an interpretation of the origin of the pseudogap phases observed there.

Results for the attractive Hubbard model.-To demonstrate the applicability of the fluctuation diagnostics, we start from a case where the underlying, dominant physics is well understood, namely, the attractive Hubbard model, $U<0$. This model captures the basic mechanisms of the BCS/Bose-Einstein crossover [68-72] and has been intensively studied both analytically and numerically, e.g., with QMC [72-74] and DMFT [75-78]. Because of the local attractive interaction, the dominant collective modes are necessarily $s$-wave pairing fluctuations $[\mathbf{Q}=(0,0)]$ in the particle channel, and, for filling $n \sim 1$, CDW fluctuations $[\mathbf{Q}=(\pi, \pi)]$ in the charge channel. As we show in the following, this underlying physics is well captured by our fluctuation diagnostics.

We present here our DCA results computed on a cluster with $N_{c}=8$ sites for a 2D Hubbard model with the following parameter set: $t=-0.25 \mathrm{eV} \quad\left(t^{\prime}=0\right)$, $U=-1 \mathrm{eV}, \mu=-0.53 \mathrm{eV}$ and $\beta=40 \mathrm{eV}^{-1}$ [51]. This leads to the occupancy $n=0.87$, for which, at this $T$, no superconducting long-range order is observed in DCA, and 

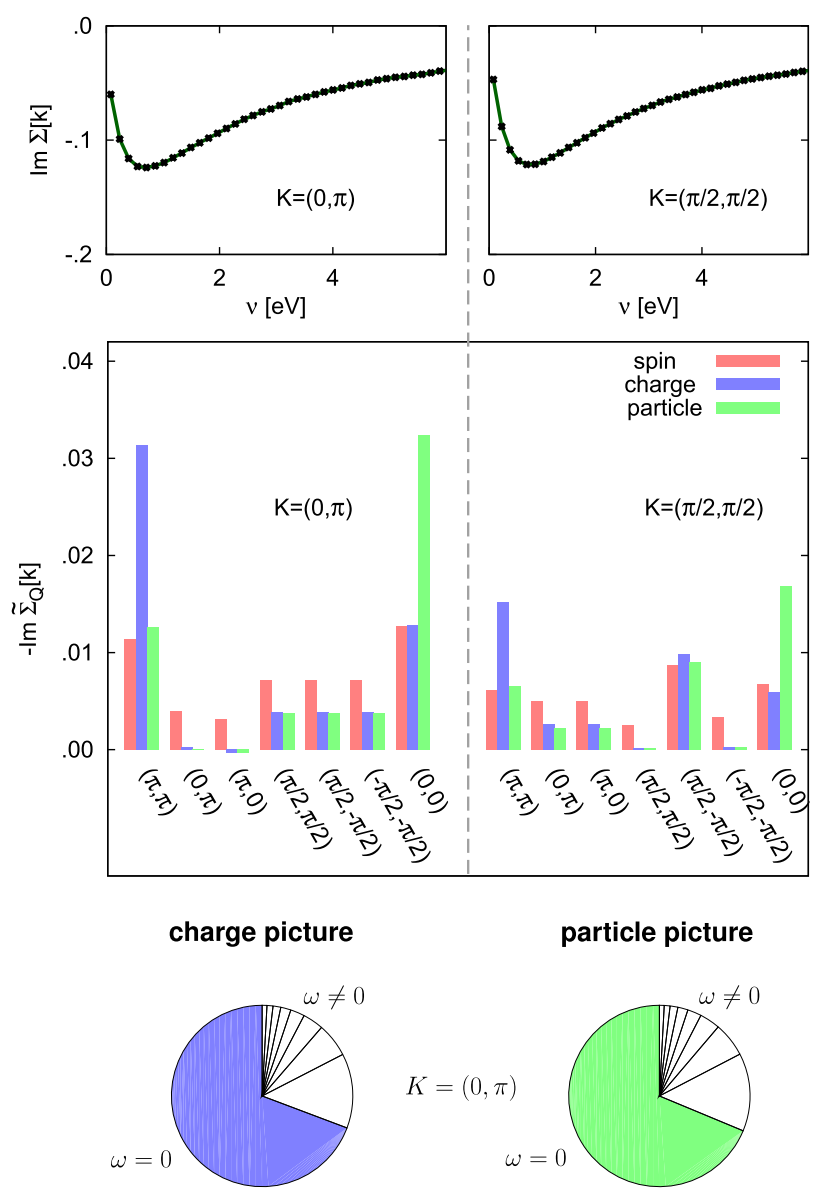

particle picture

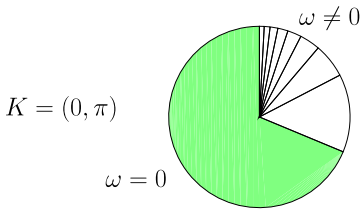

FIG. 1 (color online). Fluctuation diagnostics of $\operatorname{Im} \Sigma(\mathbf{K}, \nu)$ (first row) for the attractive Hubbard model. The histogram shows the contributions of $\operatorname{Im} \tilde{\Sigma}_{\mathbf{Q}}(\mathbf{K}, \pi / \beta)$ from different values of $\mathbf{Q}$ in the spin, charge, and particle-particle representations for the attractive 2D Hubbard model (see text). The pie charts display the relative magnitudes of $\left|\operatorname{Im} \tilde{\Sigma}_{\omega}(\mathbf{K}, \pi / \beta)\right|$ for the first eight Matsubara frequencies $|\omega|$ in the charge and particle-particle picture, respectively.

to the self-energy shown in Fig. 1 (upper panel) which exhibits a metallic behavior with weak $\mathbf{K}$ dependence. The lower panels of Fig. 1 show the fluctuation diagnostics for $\Sigma$. The histogram depicts the different contributions to Im $\Sigma[\mathbf{K}, \nu]$ for $\mathbf{K}=(0, \pi)$ and $(\pi / 2, \pi / 2)$ (upper panel of Fig. 1) at the lowest Matsubara frequency $(\nu=\pi / \beta)$ as a function of the momentum transfer $\mathbf{Q}$ within the three representations [spin, charge, and particle, i.e., via Eqs. (2), (3), (4)]. We observe large contributions for $\mathbf{Q}=(\pi, \pi)$ in the charge representation (blue bars) and for $\mathbf{Q}=(0,0)$ in the particle-particle one (green bars). At the same time, no $\mathbf{Q}$ dominates in the spin picture. Hence, the fluctuation diagnostics correctly identifies the key role of CDW and $s$-wave pairing fluctuations in this system. This outcome is supported by a complementary analysis in frequency space (pie chart in Fig. 1): Defining $\tilde{\Sigma}_{\omega}(\mathbf{K}, \nu)$ as a contribution to the self-energy, where in Eq. (1) all summations, except the one over the transfer frequency $\omega$ are performed, we observe a largely dominant contribution at $\omega=0$ $(\sim 70 \%)$ both in the charge and particle-particle pictures. This proves that the corresponding fluctuations are well defined and long lived.

Results for the repulsive Hubbard model.-We now apply the fluctuation diagnostics to the much more debated physics of the repulsive Hubbard model in two dimensions, focusing on the analysis of the pseudogap regime. As before, we use DCA calculations with a cluster of $N_{c}=8$ sites. $\Sigma$ and $F$ have been calculated using the Hirsch-Fye [79] and continuous time [80,81] QMC methods, accurately cross-checking the results. In the view of a crude modelization of the cuprate pseudogap regime, we consider the parameter set $t=-0.25 \mathrm{eV}, t^{\prime}=0.0375$, $U=1.75 \mathrm{eV}, \mu=0.6 \mathrm{eV}$ (corresponding to $n=0.94$ ) and $\beta=60 \mathrm{eV}^{-1}$ [51]. For these parameters, the selfenergy (see upper panels of Fig. 2) displays strong momentum differentiation between the "antinodal" $[\mathbf{K}=(0, \pi)]$ and the "nodal" $[\mathbf{K}=(\pi / 2, \pi / 2)]$ momentum, with a pseudogaplike behavior at the antinode $[82,83]$.

The fluctuation diagnostics is performed in Fig. 2, where we show the contributions to $\operatorname{Im} \Sigma[\mathbf{K}, \pi / \beta]$ for $\mathbf{K}=(0, \pi)$
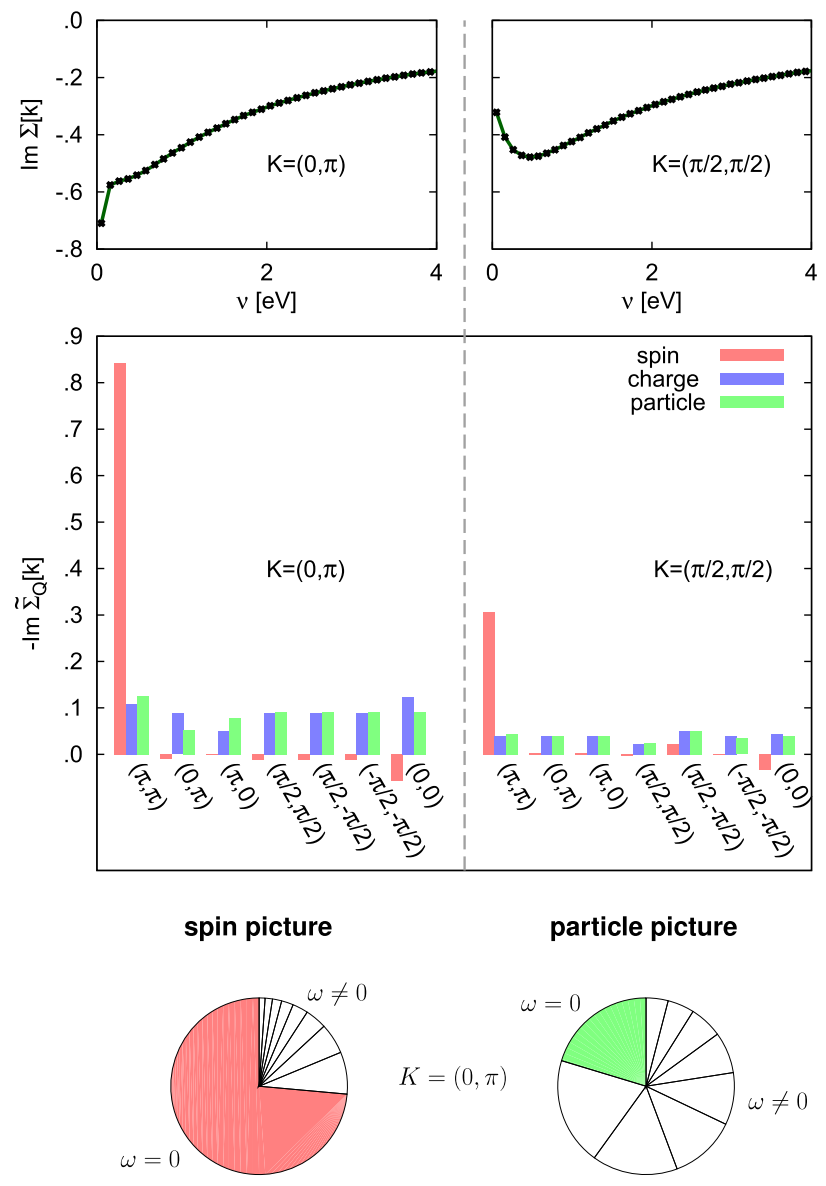

FIG. 2 (color online). As for Fig. 1: Fluctuation diagnostics of the electronic self-energy, for the case of the repulsive Hubbard model (see text and Supplemental Material [51]). 
and $(\pi / 2, \pi / 2)$ (upper panels) as a function of the transfer momentum $\mathbf{Q}$ in the three representations. This illustrates clearly the underlying physics of the pseudogap. In the spin representation (red bars in the histogram), the $\mathbf{Q}=(\pi, \pi)$ contribution dominates, and contributes more than $85 \%$ and $80 \%$ of the result for $\mathbf{K}=(0, \pi)$ and $\mathbf{K}=(\pi / 2, \pi / 2)$, respectively. Conversely, all the contributions at other transfer momenta $\mathbf{Q} \neq(\pi, \pi)$ are about an order of magnitude smaller. The dominant $\mathbf{Q}=(\pi, \pi)$ contribution is also responsible for the large momentum differentiation, being almost twice as large for the antinodal self-energy. Performing the same analysis in the charge (blue bars) or particle-particle (green bars) representation, we get a completely different shape of the histogram. In both cases, the contributions to $\Sigma$ are almost uniformly distributed among all transfer momenta $\mathbf{Q}$.

Hence, we do not find important contributions to $\Sigma$ from charge or pairing modes, while the histogram in the spinrepresentation marks the strong impact of antiferromagnetic fluctuations [6-10,84-86]. This picture is further supported by the complementary frequency analysis. The pie chart in Fig. 2 is dominated by the $\omega=0$ contribution in the spin picture, reflecting the long-lived nature of welldefined spin fluctuations. At the same time, in the particle (and charge, not shown) representation, the contributions are more uniformly distributed among all $\omega$ 's, which corresponds to short-lived pairing (charge) fluctuations. We note, eventually, that the fluctuation diagnostics of Fig. 2 qualitatively resembles the results obtained for the unfrustrated model at half-filling (not shown). In fact, a significant weakening of the spin dominance is found, moving away from the doping or interaction level, where a pseudogap is found in DCA.

Physical interpretation of the pseudogap.-We are now in the position to draw some general conclusions on the physics underlying a pseudogap. These considerations are relevant for the underdoped cuprates, up to the extent their low-energy physics is captured by the 8-site DCA for the repulsive 2D Hubbard model. We focus here on our data of Fig. 2: By means of fluctuations diagnostics we identify a well-defined $[\mathbf{Q}=(\pi, \pi)]$ collective spin mode to be responsible (on the $80 \%$ level) both for the momentum differentiation of $\Sigma$ and for its pseudogap behavior at the antinode: The large values of $\tilde{\Sigma}_{\mathbf{Q}}$ at $\mathbf{Q}=(\pi, \pi)$ and $\tilde{\Sigma}_{\omega}$ at $\omega=0$ are the distinctive hallmark of long-lived and extended (antiferromagnetic) spin fluctuations. At the same time, the rather uniform $\mathbf{Q}$ and $\omega$ distribution of $\tilde{\Sigma}_{\mathbf{Q}}$ and $\tilde{\Sigma}_{\omega}$ in the charge/particle pictures shows that the well-defined spin mode can be also viewed as short-lived and shortrange charge/pair fluctuations. The latter cannot be interpreted, hence, in terms of preformed pairs. This scenario matches very well the different estimates of fluctuation strengths in previous DCA studies [83,86,87]. We also emphasize the general applicability of our result (see Supplemental Material [51]): A well-defined mode in one channel appears as short-lived fluctuations in other channels. This dichotomy is not visible in $\Sigma$, which makes our fluctuations diagnostics a powerful tool for identifying the most convenient viewpoint to understand the physics responsible of the observed spectral properties.

Let us finally turn our attention to the still open question about the impact of superconducting $d$-wave fluctuations on the normal-state spectra in the pseudogap regime of the Hubbard model. The instantaneous fluctuations are defined as $\left\langle\Delta_{d}^{\dagger} \Delta_{d}\right\rangle$, with $\Delta_{d}^{\dagger}=\sum_{\mathbf{K}} f(\mathbf{K}) c_{\mathbf{K} \uparrow}^{\dagger} c_{-\mathbf{K} \downarrow}^{\dagger}$ and $f(\mathbf{K})=$ $\cos K_{x}-\cos K_{y}$. These $\mathbf{Q}=0$ fluctuations are certainly strong in proximity of the superconducting phase, but they were also found [83] to be significant over short distances in the pseudogap regime. Their intensity gets stronger as $U$ is increased, beyond the values where superconductivity exists. The expression for $\tilde{\Sigma}_{\mathbf{Q}=(0,0)}$ in the particle picture is closely related to $\left\langle\Delta_{d}^{\dagger} \Delta_{d}\right\rangle$, except that the factor $f(\mathbf{K})$ is missing in $\tilde{\Sigma}_{\mathbf{Q}}$ [51]. One might therefore have expected that large $\mathbf{Q}=0$ pair fluctuations, irrespectively of their lifetime, would have contributed strongly to $\Sigma$. For unconventional superconductivity, e.g., $d$ wave, this does not happen. The reason is the angular variation of $f(\mathbf{K})$. For strong pair fluctuations, the variations of $f(\mathbf{K})$ make the contributions to the fluctuations add up, while the contributions to $\Sigma$ then tend to cancel. This explains why suppressing superconductivity fluctuations $[42,44,83,87-91]$ does not affect the description of the pseudogap of the Hubbard model. In the case of a purely local interaction such as in the EOM like Eq. (1), enhanced $\left\langle\Delta_{d}^{\dagger} \Delta_{d}\right\rangle$ fluctuations are mostly averaged out by the momentum summation (see Supplemental Material [51]).

Our diagnosis of dominant spin fluctuations in the DCA self-energy in the underdoped 2D-Hubbard model does not represent per se the conclusive scenario for the cuprate pseudogap. However, important information about the realistic modeling of cuprates can be already extracted: If definitive experimental evidence for an impact of supposedly "secondary" (e.g., charge) fluctuations on the pseudogap is found, extensions of the modelization will be unavoidable for a correct pseudogap theory: Nonlocal interactions (e.g., extended Hubbard model) or explicit inclusion of the oxygen orbitals (e.g., Emery model) might be required. In fact, such extensions represent in itself an intriguing playground for future fluctuation diagnostics applications.

Conclusions.-We have shown that if a simultaneous calculation of the self-energy and the vertex functions is performed, it is possible to identify the impact of the different collective modes on the spectra of correlated systems (fluctuation diagnostics). This is achieved by expressing the equation of motion for $\Sigma$ in different representations (e.g., spin, charge, or particle), which avoids all the intrinsic instabilities of parquet decompositions. We apply this procedure to the $U<0$ and $U>0$ 2D Hubbard model. In the attractive case we have confirmed 
the dominant role of pair fluctuations, supporting the validity of our approach. For the repulsive model, relevant for the physics of the underdoped cuprates, spin fluctuations emerged as mainly responsible for the spectral function results, in agreement with other studies $[6-10,86]$. The same well-defined spin modes might appear, on a different perspective, as strong, but rapidly decaying, pair fluctuations. Finally, for a purely local interaction, $d$-wave pairing fluctuations will only weakly affect the pseudogap spectral properties even on the verge of the superconducting transition.

These results, as well as the insight on the pseudogap physics, suggest that fluctuation diagnostics can be broadly used in future studies. The progress in calculating vertex functions $[66,92,93]$ will allow its applicability also to other, more complex, multiorbital models [94-100]: Here, due to the increased number of degrees of freedom, the identification of the dominant fluctuation mode(s) will be of the utmost importance for a correct physical understanding.

We thank A. Tagliavini, C. Taranto, S. Andergassen, M. Sing, and M. Capone for insightful discussions. We acknowledge support from FWF through the $\mathrm{PhD}$ School "Building Solids for Function" (T. S., Project No. W1243) and the project I 610 (G. R., A. T.), from the research unit FOR 1346 of the DFG (G.S.), from MINECO: MAT2012-37263-C02-01 (J. M.), and from the Simons Foundation (J. P. F. L., E. G.). G. S. and A. T. also acknowledge the hospitality in Campello sul Clitunno.

[1] A. Damascelli, Z. Hussain, and Zhi-Xun Shen, Rev. Mod. Phys. 75, 473 (2003).

[2] G. Binnig and H. Rohrer, Rev. Mod. Phys. 59, 615 (1987).

[3] O. Fischer, M. Kugler, I. Maggio-Aprile, C. Berthod, and C. Renner, Rev. Mod. Phys. 79, 353 (2007).

[4] T. Timusk and B. W. Statt, Rep. Prog. Phys. 62, 61 (1999).

[5] M. Uchida et al., Phys. Rev. Lett. 106, 027001 (2011).

[6] D. J. Scalapino, Rev. Mod. Phys. 84, 1383 (2012).

[7] C. Huscroft, M. Jarrell, Th. Maier, S. Moukouri, and A. N. Tahvildarzadeh, Phys. Rev. Lett. 86, 139 (2001).

[8] D. Sénéchal and A. M. S. Tremblay, Phys. Rev. Lett. 92, 126401 (2004).

[9] B. Kyung, S. S. Kancharla, D. Senechal, A. M. S. Tremblay, M. Civelli, and G. Kotliar, Phys. Rev. B 73, 165114 (2006).

[10] A. Macridin, M. Jarrell, T. Maier, P. R. C. Kent, and E. D’Azevedo, Phys. Rev. Lett. 97, 036401 (2006).

[11] V. J. Emery and S. A. Kivelson, Nature (London) 374, 434 (1995).

[12] Z. A. Xu, N. P. Ong, Y. Wang, T. Kakeshita, and S. Uchida, Nature (London) 406, 486 (2000).

[13] Y. Wang, L. Li, M. J. Naughton, G. D. Gu, S. Uchida, and N. P. Ong, Phys. Rev. Lett. 95, 247002 (2005).

[14] Y. Kosaka, T. Hanaguri, M. Azuma, M. Takano, J. C. Davis, and H. Takagi, Nat. Phys. 8, 534 (2012).
[15] V. Mishra, U. Chatterjee, J. C. Campuzano, and M. R. Norman, Nat. Phys. 10, 357 (2014).

[16] T. D. Stanescu and P. Phillips, Phys. Rev. Lett. 91, 017002 (2003).

[17] M. Imada, S. Sakai, Y. Yamaji, and Y. Motome, J. Phys. Conf. Ser. 449, 012005 (2013).

[18] E. H. da Silva Neto, P. Aynajian, A. Frano, R. Comin, E. Schierle, E. Weschke, A. Gyenis, J. Wen, J. Schneeloch, Z. $\mathrm{Xu}, \mathrm{S}$. Ono, G. Gu, M. Le Tacon, and A. Yazdani, Science 343, 393 (2014).

[19] K.-Y. Yang, T. M. Rice, and F. C. Zhang, Phys. Rev. B 73, 174501 (2006).

[20] T. M. Rice, K.-Y. Yang, and F. C. Zhang, Rep. Prog. Phys. 75, 016502 (2012).

[21] R. Comin et al., Science, 343, 390 (2014).

[22] S. I. Mirzaei, D. Strickera, J. N. Hancocka, C. Berthoda, A. Georges, E. van Heumen, M. K. Chan, X. Zhao, Y. Li, M. Greven, N. Barisiĉ, and D. van der Marel, Proc. Natl. Acad. Sci. U.S.A. 110, 5774 (2013).

[23] R. J. Birgeneau, C. Stock, J. M. Tranquada, and K. Yamada, J. Phys. Soc. Jpn. 75, 111003 (2006).

[24] D. N. Basov and T. Timusk, Rev. Mod. Phys. 77, 721 (2005).

[25] F. Cilento et al., Nat. Commun. 5, 4353 (2014).

[26] J. Sonier, J. Brewer, and R. Kiefl, Rev. Mod. Phys. 72, 769 (2000).

[27] F. O. Schumann, J. Kirschner, and J. Berakdar, Phys. Rev. Lett. 95, 117601 (2005); F. O. Schumann, C. Winkler, and J. Kirschner, Phys. Rev. Lett. 98, 257604 (2007).

[28] I. S. Brandt, Z. Wei, F. O. Schumann, and J. Kirschner, Phys. Rev. Lett. 113, 107601 (2014).

[29] F. O. Schumann, L. Behnke, C.-H. Li, and J. Kirschner, J. Phys. Condens. Matter 25, 094002 (2013).

[30] R. Blankenbecler, D. J. Scalapino, and R. L. Sugar, Phys. Rev. D 24, 2278 (1981).

[31] W. Metzner, M. Salmhofer, C. Honerkamp, V. Meden, and K. Schönhammer, Rev. Mod. Phys. 84, 299 (2012).

[32] N. E. Bickers, D. J. Scalapino, and S. R. White, Phys. Rev. Lett. 62, 961 (1989).

[33] V. Janiŝ, J. Phys. Condens. Matter 10, 2915 (1998); Phys. Rev. B 60, 11345 (1999).

[34] S. X. Yang, H. Fotso, J. Liu, T. A. Maier, K. Tomko, E. F. D'Azevedo, R. T. Scalettar, T. Pruschke, and M. Jarrell, Phys. Rev. E 80, 046706 (2009).

[35] T. Maier, M. Jarrell, T. Pruschke, and M. H. Hettler, Rev. Mod. Phys. 77, 1027 (2005).

[36] W. Metzner and D. Vollhardt, Phys. Rev. Lett. 62, 324 (1989).

[37] A. Georges, G. Kotliar, W. Krauth, and M. Rozenberg, Rev. Mod. Phys. 68, 13 (1996).

[38] A. I. Lichtenstein and M. I. Katsnelson, Phys. Rev. B 62 , R9283 (2000).

[39] G. Kotliar, S. Y. Savrasov, G. Pálsson, and G. Biroli, Phys. Rev. Lett. 87, 186401 (2001).

[40] M. H. Hettler, M. Mukherjee, M. Jarrell, and H. R. Krishnamurthy, Phys. Rev. B 61, 12739 (2000).

[41] A. Toschi, A. A. Katanin, and K. Held, Phys. Rev. B 75, 045118 (2007).

[42] K. Held, A. A. Katanin, and A. Toschi, Prog. Theor. Phys. Suppl. 176, 117 (2008). 
[43] A. N. Rubtsov, M. I. Katsnelson, and A. I. Lichtenstein, Phys. Rev. B 77, 033101 (2008).

[44] H. Hafermann, G. Li, A. N. Rubtsov, M. I. Katsnelson, A. I. Lichtenstein, and H. Monien, Phys. Rev. Lett. 102, 206401 (2009).

[45] C. Slezak, M. Jarrell, Th. Maier, and J. Deisz, J. Phys. Condens. Matter 21, 435604 (2009).

[46] A. Valli, G. Sangiovanni, O. Gunnarsson, A. Toschi, and K. Held, Phys. Rev. Lett. 104, 246402 (2010).

[47] G. Rohringer, A. Toschi, H. Hafermann, K. Held, V. I. Anisimov, and A. A. Katanin, Phys. Rev. B 88, 115112 (2013).

[48] S.-X. Yang, H. Fotso, H. Hafermann, K.-M. Tam, J. Moreno, T. Pruschke, and M. Jarrell, Phys. Rev. B 84, 155106 (2011).

[49] C. Taranto, S. Andergassen, J. Bauer, K. Held, A. Katanin, W. Metzner, G. Rohringer, and A. Toschi, Phys. Rev. Lett. 112, 196402 (2014).

[50] A. Valli, T. Schäfer, P. Thunström, G. Rohringer, S. Andergassen, G. Sangiovanni, K. Held, and A. Toschi, Phys. Rev. B 91, 115115 (2015).

[51] See Supplemental Material http://link.aps.org/ supplemental/10.1103/PhysRevLett.114.236402 for further algorithmic details and an analysis of limiting cases, which includes Refs. [52-55].

[52] G. Rohringer, PhD thesis, TU Wien, 2013.

[53] M. Jarrell, Th. Maier, C. Huscroft, and S. Moukouri, Phys. Rev. B 64, 195130 (2001).

[54] C. Karrasch, R. Hedden, R. Peters, Th. Pruschke, K. Schönhammer, and V. Meden, J. Phys. Condens. Matter 20, 345205 (2008).

[55] C. Husemann and M. Salmhofer, Phys. Rev. B 79, 195125 (2009).

[56] A. A. Abrikosov et al., Methods of Quantum Field Theory in Statistical Physics (Dover, New York, 1963).

[57] J. Hubbard, Proc. R. Soc. A 276, 238 (1963).

[58] M. C. Gutzwiller, Phys. Rev. Lett. 10, 159 (1963).

[59] J. Kanamori, Prog. Theor. Phys. 30, 275 (1963).

[60] D. Senechal et al., Theoretical Methods for Strongly Correlated Electrons (Springer, Berlin, 2003), Chap. 6.

[61] T. Schäfer, G. Rohringer, O. Gunnarsson, S. Ciuchi, G. Sangiovanni, and A. Toschi, Phys. Rev. Lett. 110, 246405 (2013).

[62] See unpublished Appendix of Ref. [48] in arXiv:1104.2854v1.

[63] V. Janiš and V. Pokorný, Phys. Rev. B 90, 045143 (2014).

[64] E. Kozik, M. Ferrero, and A. Georges, Phys. Rev. Lett. 114, 156402 (2015).

[65] O. Gunnarsson, T. Schäfer, J. P. F. LeBlanc, J. Merino, G. Sangiovanni, G. Rohringer, and A. Toschi (to be published).

[66] G. Rohringer, A. Valli, and A. Toschi, Phys. Rev. B 86, 125114 (2012).

[67] K. M. Tam, H. Fotso, S. X. H. Yang, and T. W. Lee, J. Moreno, J. Ramanujam, and M. Jarrell, Phys. Rev. E, 87, 013311 (2013).

[68] R. Micnas, J. Ranninger, and S. Robaszkiewicz, Rev. Mod. Phys. 62, 113 (1990).

[69] C. A. R. Sà de Melo, M. Randeria, and J. R. Engelbrecht, Phys. Rev. Lett. 71, 3202 (1993).
[70] R. Haussmann, Z. Phys. B 91, 291 (1993).

[71] F. Pistolesi and G. C. Strinati, Phys. Rev. B 53, 15168 (1996).

[72] B. Kyung, S. Allen, and A.-M. S. Tremblay, Phys. Rev. B 64, 075116 (2001).

[73] A. Moreo and D. J. Scalapino, Phys. Rev. Lett. 66, 946 (1991).

[74] T. Paiva, R. R. dos Santos, R. T. Scalettar, and P. J. H. Denteneer, Phys. Rev. B 69, 184501 (2004).

[75] M. Keller, W. Metzner, and U. Schollwöck, Phys. Rev. Lett. 86, 4612 (2001).

[76] A. Toschi, P. Barone, M. Capone, and C. Castellani, New J. Phys. 7, 7 (2005).

[77] A. Toschi, M. Capone, and C. Castellani, Phys. Rev. B 72, 235118 (2005).

[78] A. Garg, H. R. Krishnamurthy, and M. Randeria, Phys. Rev. B 72, 024517 (2005).

[79] J. E. Hirsch and R. M. Fye, Phys. Rev. Lett. 56, 2521 (1986).

[80] E. Gull, P. Werner, O. Parcollet, and M. Troyer, Eur. Phys. Lett. 82, 57003 (2008).

[81] E. Gull, A. J. Millis, A. I. Lichtenstein, A. N. Rubtsov, M. Troyer, and P. Werner, Rev. Mod. Phys. 83, 349 (2011).

[82] E. Gull, M. Ferrero, O. Parcollet, A. Georges, and A. J. Millis, Phys. Rev. B 82, 155101 (2010).

[83] J. Merino and O. Gunnarsson, Phys. Rev. B 89, 245130 (2014); see also: arXiv:1310.4597.

[84] D. Rohe and W. Metzner, Phys. Rev. B 71, 115116 (2005).

[85] E. Gull, O. Parcollet, P. Werner, and A. J. Millis, Phys. Rev. B 80, 245102 (2009).

[86] E. Gull and A. J. Millis, Phys. Rev. B 86, 241106(R) (2012).

[87] J. Merino and O. Gunnarsson, J. Phys. Condens. Matter 25, 052201 (2013).

[88] Y. M. Vilk and A. M. S. Tremblay, J. Phys. I 7, 1309 (1997).

[89] E. Z. Kuchinskii, I. A. Nekrasov, and M. V. Sadovskii, Sov. Phys. JETP Lett. 82, 217 (2005).

[90] A. A. Katanin, A. Toschi, and K. Held, Phys. Rev. B 80, 075104 (2009).

[91] A. Avella, Adv. Condens. Matter Phys. 2014, 515698 (2014).

[92] O. Gunnarsson, G. Sangiovanni, A. Valli, and M. W. Haverkort, Phys. Rev. B 82, 233104 (2010).

[93] H. Hafermann, Phys. Rev. B 89, 235128 (2014).

[94] S. Biermann, A. Poteryaev, A. I. Lichtenstein, and A. Georges, Phys. Rev. Lett. 94, 026404 (2005).

[95] T. Saha-Dasgupta, S. Glawion, M. Sing, R. Claessen, and R. Valentí, New J. Phys. 9, 380 (2007).

[96] J. M. Tomczak, F. Aryasetiawan, and S. Biermann, Phys. Rev. B 78, 115103 (2008).

[97] M. Aichhorn, T. Saha-Dasgupta, R. Valentí, S. Glawion, M. Sing, and R. Claessen, Phys. Rev. B 80, 115129 (2009).

[98] A. Toschi, G. Rohringer, A. A. Katanin, and K. Held, Ann. Phys. (Amsterdam) 523, 698 (2011).

[99] C. Platt, W. Hanke, and R. Thomale, Adv. Phys. 62, 453 (2013).

[100] Y. Nomura, S. Sakai, and R. Arita, Phys. Rev. B 89, 195146 (2014). 